\title{
A Maximum Likelihood Fine Timing Estimation for Wireless OFDM Systems
}

\author{
Hao Zhou and Yih-Fang Huang, Fellow, IEEE
}

\begin{abstract}
Orthogonal Frequency Division Multiplexing (OFDM) systems are more sensitive to timing synchronization than single carrier systems. This paper presents a fine timing synchronization scheme which utilizes the channel impulse response (CIR) estimated from frequency-domain samples at OFDM receivers. A Maximum Likelihood Estimate (MLE) of timing offset is derived based on the probability distribution of the estimated CIR under time-varying multipath fading. The ML timing scheme is further developed for both integer precision and real-valued precision implementations. In the real-valued timing precision case, a delay locked loop (DLL) structure is devised as an effective way to implement the MLE. Both analysis and simulations of the proposed MLE showed significant improvement over existing schemes under time-varying multipath fading channels.
\end{abstract}

Index Terms-Maximum likelihood estimate, OFDM, time-varying multipath fading channel, timing synchronization.

\section{INTRODUCTION}

$\mathbf{O}$ RTHOGONAL frequency division multiplexing (OFDM) has become a popular technique for high-bit-rate wireless communications [1], [2]. High spectral efficiency and efficient multipath immunity are two major features of this technology. However, compared to single-carrier systems, OFDM systems are more susceptible to synchronization errors, specifically, symbol timing and carrier frequency errors [3]-[6].

This paper considers the problem of symbol timing in OFDM systems. Timing errors in wireless OFDM systems, like those in other wireless systems, arise from the frequency difference between the transceiver oscillators as well as the Doppler effect due to the transceiver mobility. Timing offset has two major effects in OFDM systems: it causes extra interference (namely, intersymbol interference (ISI) and interchannel interference (ICI)) which degrades the performance of channel estimation [6]. If the samples contained in one received OFDM symbol is influenced by more than one transmitted OFDM symbol, the demodulated signal will be corrupted by ISI and the orthogonality between subcarriers can also be violated, thereby causing ICI [6], [7]. Both ISI and ICI cause additional disturbance and distortion to the demodulated signal. Thus they may result in signif-

Manuscript received October 15, 2007; revised August 29, 2008. Current version published February 25, 2009.

H. Zhou is with Atheros Communications, Santa Clara, CA 95054-3644, USA (e-mail: haozhou_sh@hotmail.com).

Y.-F. Huang is with the Department of Electrical Engineering, University of Notre Dame, Notre Dame, IN 46556, USA (e-mail: huang@nd.edu).

Color versions of one or more of the figures in this paper are available online at http://ieeexplore.ieee.org.

Digital Object Identifier 10.1109/TBC.2008.2007457 icant performance degradation. In a coherent OFDM system, timing offset has another, possibly even more severe, impact on system performance due to its adverse effect on channel estimation [6], [8], [10], [12]. In practice, when some portions of the effective channel are shifted outside the channel estimation window due to timing offsets, the channel estimates will suffer additional errors [6]. This error effect is more pronounced for high-performance channel estimators which usually have a narrow channel estimation window matched to the channel impulse response (CIR) [6], [12]. Such estimators include the FFTbased optimum interpolator [10], the minimum mean-squareerror (MMSE) channel estimator [12] and the enhanced channel estimators based on a reduced signal space [13]-[15]. All these high-performance channel estimators demand a high precision timing recovery at the receiver.

Timing synchronization for OFDM systems usually takes two steps. First, a coarse synchronization acquires a rough fast Fourier Transform (FFT) window position in the received OFDM signal stream. This is followed by fine timing synchronization which refines the timing position of the FFT window and continues tracking the symbol timing throughout the entire data transmission [16], [17]. The task of fine timing can be implemented using cross-correlation of the training sequence in the time domain [18]. However, for multipath channels, those time-domain correlation methods in general can not guarantee sufficiently accurate timing positions[17], because the multipath results in interpath interference in the time-domain correlation. In [16], [17], [19]-[22], frequency-domain schemes are proposed to provide better timing synchronization for multipath fading channels. These schemes first estimate the CIR, then find the timing position by locating the estimated CIR inside the estimation window.

A frequency-domain delay-locked-loop (DLL) tracking structure is proposed in [17]. It is shown to be equivalent to peak-finding in the estimated CIR and it provides a maximum likelihood (ML) solution for channels with additive white Gaussian noise (AWGN). In [19], a frequency-domain algorithm is presented to find the first channel path in the estimated CIR with the aid of two SNR-based thresholds. In [16], [21], [22], maximizing the CIR energy in a rectangular window is proposed as an alternative approach to refine the timing position. Thanks to their ability to resolve channel multipaths, these frequency-domain schemes are generally superior to the time-domain correlation methods. However, when multipath channels experience time-varying fading, those schemes may still have degraded tracking performance due to the instantaneous energy variation of individual paths. The resulting tracking error could impair the performance of those systems using high-performance channel estimates [9]-[15]. 
In this paper, we propose a frequency-domain fine timing scheme, which utilizes channel statistical information to further improve the timing performance for time-varying fading channels [23]. The CIR estimated at the receiver is modeled as a vector complex Gaussian random process, parameterized by the timing offset. An ML estimate (MLE) of the timing offset is derived based on this statistical model. The resulting MLE is further developed for both integer- and real-valued timing precision implementation. For the integer precision, the MLE is simplified to be implemented as a power-delay-profile (PDP)-based correlation scheme. Here, we use the correct estimation probability as a measure to compare the performance of the proposed MLE with that of the other schemes. For the real-valued timing precision, the proposed scheme is developed into a PDP-based DLL structure to track the fractional timing offset. Theoretical analysis and numerical simulations show that, in both cases, the tracking ability of the proposed scheme is significantly better (measured by the mean-squared error) than those of the existing methods [17], [19], [21], [22]. This improved tracking ability is very useful for the advanced OFDM systems under time-varying fading channels. In particular, it provides an accurate timing to those systems that employ high-performance channel estimators [13]-[15]. As an example, it is shown by simulation that the improved timing actually helps retain the optimum performance of the OFDM systems that employ MMSE channel estimation and provides a system performance gain over the conventional systems that use the ML channel estimation.

\section{SYSTEM MODEL}

We start by briefly reviewing the OFDM system with an emphasis on the timing issue. A wireless channel is commonly formulated as

$$
h(t, \tau)=\sum_{l=1}^{L} h_{l}(t) \delta\left(\tau-\tau_{l}\right)
$$

where $h_{l}(t)$ is the $l$ th complex path gain which is usually modeled as an independent complex Gaussian process with variance $\sigma_{h_{l}}^{2}, \tau_{l}$ is the time delay of the $l$ th path [2] and $L$ is the number of independent multipaths.

An OFDM signal is generated by performing an inverse FFT (IFFT) on $N$ complex valued data (constellation points) $\left\{X_{i, n}, n=-N / 2, \ldots, N / 2-1\right\} \mathrm{d}$, resulting in a block of $N$ time-domain samples. Here, $N$ is usually a power of two for efficient FFT processing. The last $N_{c p}$ samples of each signal block are added to the front of the $N$ samples as a cyclic-prefix, and all $N_{c p}+N$ samples constitute an entire OFDM symbol. The modulated OFDM signal $x(t)$ can be expressed as

$$
x(t)=\sum_{n=-N / 2}^{N / 2-1} X_{n} \Phi_{n}(t), \quad \text { for } 0 \leq t<T_{s}
$$

where $\Phi_{n}(t)$ is the subcarrier modulation pulse, i.e.,

$$
\begin{aligned}
& \Phi_{n}(t)=e^{j 2 \pi\left(n / T_{u}\right)\left(t-T_{g}\right)}, \\
& \qquad \text { for } 0 \leq t<T_{s},-N / 2 \leq n \leq N / 2-1
\end{aligned}
$$

in (3), $T$ is the sampling time duration, $T_{u}(=N T)$ is the effective OFDM symbol duration, $T_{g}\left(=N_{c p} T\right)$ is the guard interval duration, and $T_{s}=T_{u}+T_{g}=\left(N+N_{c p}\right) T$ is the duration of an entire OFDM symbol.

After the modulated signal $x(t)$ passes through the wireless channel (1), the receiver samples the waveform and recovers the original data through FFT processing. Timing offsets usually occur when the received OFDM symbols are sampled. If the symbol timing position begins within the region $\left\{\tau_{\max }, T_{g}\right\}$, i.e., the symbol timing error $e_{\xi}$ is within the ISI-free region of the cyclic-prefix $\left(-N_{c p}+\tau_{\max } / T \leq e_{\xi} \leq 0\right)$, the received signal would be

$$
\begin{aligned}
y(t & \left.=k T+T_{g}+e_{\xi} T\right) \\
& =\sum_{l=1}^{L} h_{l} \cdot x\left(k T+T_{g}+e_{\xi} T-\tau_{l}\right)+n_{k} \\
& =\sum_{l=1}^{L} h_{l} \sum_{n=-N / 2}^{N / 2-1} X_{n} \Phi_{n}\left(k T+T_{g}+\left(e_{\xi}-\tau_{l} / T\right) T\right)+n_{k} \\
& =\sum_{n=-N / 2}^{N / 2-1}\left[X_{n} \sum_{l=1}^{L} h_{l} \cdot e^{j \frac{2 \pi n\left(e_{\xi}-\tau_{l} / T\right)}{N}}\right] e^{j \frac{2 \pi n k}{N}}+n_{k}
\end{aligned}
$$

where $n_{k}$ is a complex AWGN with variance $\sigma^{2}$ and $\tau_{\max }=$ $\max _{l}\left\{\tau_{l}\right\}$. Here, we assume that the channel multipath $h_{l}(t)$ remains constant within one OFDM symbol, thus the parameter $t$ can be omitted for simplicity.

Therefore, the FFT output of the OFDM symbol, observed from (4), is

$$
\begin{aligned}
Y_{n} & =X_{n} \cdot \sum_{l=1}^{L} h_{l} \cdot e^{j \frac{2 \pi n\left(e_{\xi}-\tau_{l} / T\right)}{N}}+N_{n} \\
& =X_{n} \cdot \tilde{H}_{n}\left(e_{\xi}\right)+N_{n}, \quad \text { for }-N / 2 \leq n \leq N / 2-1
\end{aligned}
$$

where $\tilde{H}_{n}\left(e_{\xi}\right)=\left(\sum_{l=1}^{L} h_{l} \cdot e^{j\left(-2 \pi n \tau_{l} / T / N\right)}\right) e^{j\left(2 \pi n e_{\xi} / N\right)}$, and $N_{n}$ (the FFT of $n_{k}$ ) is a complex AWGN with variance $N \sigma^{2}$. Denote the effective channel transfer function (CTF) as a vector $\tilde{\mathbf{H}}\left(e_{\xi}\right)=\left\{\tilde{H}_{n}\left(e_{\xi}\right), n=-N / 2, \ldots, N / 2-1\right\}$. The effective $\mathrm{CTF}$ can be expressed as

$$
\tilde{\mathbf{H}}\left(e_{\xi}\right)=\mathbf{M}\left(e_{\xi}\right) \mathbf{h}
$$

where $\mathbf{M}\left(e_{\xi}\right)$ is an $N \times L$ matrix whose $(n, l)$-th element is equal to $e^{j 2 \pi n\left(e_{\xi}-\tau_{l} / T\right) / N}$, and $\mathbf{h}$, which contains the amplitude information of the channel, is an $L \times 1$ vector whose $l$-th element is equal to $h_{\boldsymbol{l}}(1)$. Here, $\mathbf{M}\left(e_{\xi}\right)$ is the modeling matrix that comprises the inherent time delay relationship $\left(\tau_{l}\right)$ among the uncorrelated multipaths and the channel timing offset information $\left(e_{\xi}\right)$. Note that $\mathbf{M}\left(e_{\xi}\right)$ is used only for the purpose of analysis and it is not required in implementing the integer and the real-valued precision timing synchronizers that are to be proposed in the following sections.

If the timing offset exceeds the ISI-free region, the orthogonality among the subcarriers may be destroyed. The resulting FFT output of the OFDM symbol would appear as [6], [22]

$$
Y_{n}=\alpha\left(e_{\xi}\right) \cdot X_{n} \cdot \tilde{H}_{n}\left(e_{\xi}\right)+I_{n}+N_{n}
$$


where

$$
\alpha\left(e_{\xi}\right) \cong \sum_{l=1}^{L}\left|h_{l}\right|^{2} \frac{N-\Delta e_{l}}{N}
$$

and $I_{n}$ is a term that accounts for both ISI and ICI which is modeled as a Gaussian random variable with variance [6]

$$
\sigma_{e}^{2}=\sum_{l=1}^{L}\left|h_{l}\right|^{2}\left[2 \frac{\Delta e_{l}}{N}-\left(\frac{\Delta e_{l}}{N}\right)^{2}\right]
$$

where for $e_{\xi}>0$

$$
\Delta e_{l}= \begin{cases}e_{\xi}-\tau_{l} / T, & e_{\xi}>\tau_{l} / T \\ \tau_{l} / T-N_{c p}-e_{\xi}, & 0<e_{\xi}<-N_{c p}+\tau_{l} / T \\ 0, & \text { else }\end{cases}
$$

or for $e_{\xi}<-N_{c p}+\tau_{\max } / T$,

$$
\Delta e_{l}= \begin{cases}\tau_{l} / T-N_{c p}-e_{\xi}, & 0<e_{\xi}<-N_{c p}+\tau_{l} / T \\ 0, & \text { else }\end{cases}
$$

As mentioned in [6], for OFDM systems with a large $N$, the attenuation $\alpha\left(e_{\xi}\right)$ can be neglected. By combining ISI and ICI together with the additive Gaussian noise, we can formulate the frequency domain OFDM symbol as

$$
Y_{n} \cong X_{n} \cdot \tilde{H}_{n}\left(e_{\xi}\right)+Z_{n}
$$

and (5) can be seen as a special case of (12) with $I_{n}$ being zero. Without loss of generality, we assume that $Z_{n}$ in (12) is a zeromean Gaussian random variable. Accordingly, the MLE of the $\operatorname{CTF}\left(\tilde{H}_{n}\left(e_{\xi}\right)\right)$ is simply

$$
\hat{H}_{n}\left(e_{\xi}\right)=Y_{n} / X_{n}=\tilde{H}_{n}\left(e_{\xi}\right)+Z_{n} / X_{n}
$$

which is equivalent to the least-squares (LS) channel estimate given in [2], [24]. The estimated CIR $\hat{\mathbf{h}}=\left\{\hat{h}_{k}\right\}$ is obtained by the IFFT of the estimated CTF $\hat{\mathbf{H}}=\left\{\hat{H}_{n}\right\}$ as

$$
\begin{aligned}
\hat{\mathbf{h}}\left(e_{\xi}\right) & =\frac{1}{N} \mathbf{W}^{H} \hat{\mathbf{H}}\left(e_{\xi}\right) \\
& =\underbrace{\frac{1}{N} \mathbf{W}^{H} \mathbf{M}\left(e_{\xi}\right) \mathbf{h}}_{\text {I }}+\underbrace{\frac{1}{N} \mathbf{W}^{H} \mathbf{X}^{-1} \mathbf{Z}}_{\text {II }}
\end{aligned}
$$

where $\mathbf{W}$ is the $N \times N$ FFT matrix with $[\mathbf{W}]_{k n}=e^{-j 2 \pi k n / N}$, $\mathbf{X}$ is the diagonal data matrix with diagonal entries $[\mathbf{X}]_{n, n}$ equal to $X_{n}$, and $\mathbf{Z}$ is the noise vector consisting of $Z_{n}$. From (14), we notice that the estimated CIR, $\hat{\mathbf{h}}\left(e_{\xi}\right)$, is a time-shifted interpolation $\left[(1 / N) \mathbf{W}^{H} \mathbf{M}\left(e_{\xi}\right)\right]$ of the true channel $\mathbf{h}$ corrupted by Gaussian noise. By assuming a complex Gaussian channel model for $\mathbf{h}$, the probability density function of $\hat{\mathbf{h}}\left(e_{\xi}\right)$ is shown to be

$$
\begin{aligned}
& P\left(\hat{\mathbf{h}}\left(e_{\xi}\right) ; e_{\xi}\right)=\frac{1}{\pi^{N}\left|\operatorname{det}\left(\mathbf{C}_{\mathbf{n}}\left(e_{\xi}\right)\right)\right|} \\
& \times \exp \left[-\hat{\mathbf{h}}^{H}\left(e_{\xi}\right) \mathbf{C}_{\mathbf{n}}^{-1}\left(e_{\xi}\right) \hat{\mathbf{h}}\left(e_{\xi}\right)\right]
\end{aligned}
$$

where $\mathbf{C}_{\mathbf{n}}\left(e_{\xi}\right)$ is the autocorrelation matrix of the estimated $\operatorname{CIR}\left(\hat{\mathbf{h}}\left(e_{\xi}\right)\right)$, i.e., $\mathbf{C}_{\mathbf{n}}\left(e_{\xi}\right)=E\left[\hat{\mathbf{h}}\left(e_{\xi}\right) \hat{\mathbf{h}}\left(e_{\xi}\right)^{H}\right]$. According to the channel (I) and the noise (II) terms in (14), $\mathbf{C}_{\mathbf{n}}\left(e_{\xi}\right)$ can be expressed as

$$
\begin{aligned}
\mathbf{C}_{\mathbf{n}}\left(e_{\xi}\right)= & \underbrace{\frac{1}{N^{2}} \mathbf{W}^{H} \mathbf{M}\left(e_{\xi}\right) E\left[\mathbf{h h}^{H}\right] \mathbf{M}\left(e_{\xi}\right)^{H} \mathbf{W}}_{\text {I:C(e } \left.\mathbf{e}_{\xi}\right)} \\
& +\underbrace{\frac{1}{N^{2}} \mathbf{W}^{H} \mathbf{X}^{-1} E\left[\mathbf{Z} \mathbf{Z}^{H}\right]\left[\mathbf{X}^{-1}\right]^{H} \mathbf{W}}_{\text {II }} \\
\approx & \underbrace{\frac{1}{N^{2}} \mathbf{W}^{H} \mathbf{M}\left(e_{\xi}\right) \mathbf{D}_{L} \mathbf{M}\left(e_{\xi}\right)^{H} \mathbf{W}}_{\text {I:C }\left(\mathbf{e}_{\xi}\right)}+\underbrace{\frac{\beta}{N \gamma} \mathbf{I}}_{\text {II }}
\end{aligned}
$$

where $\mathbf{D}_{L}$ is an $L \times L$ diagonal matrix with diagonal elements $\sigma_{h_{l}}^{2}, \beta=E\left\{|X|^{2}\right\} E\left\{1 /|X|^{2}\right\}$, and $\gamma=E\left\{|X|^{2}\right\} /\left(N \sigma^{2}+\sigma_{e}^{2}\right)$ is the signal to interference and noise ratio (SINR) at the receiver. Equation (17) is obtained by approximating the noise term (II) with $\mathbf{X}^{-1}\left[\mathbf{X}^{-1}\right]^{H} \approx E\left\{1 /|X|^{2}\right\} \mathbf{I}$ and $E\left[\mathbf{Z Z}^{H}\right] \approx$ $\left(N \sigma^{2}+\sigma_{e}^{2}\right) \mathbf{I}$. It is easily seen from (17) that the estimated channel autocorrelation $\mathbf{C}_{\mathbf{n}}\left(e_{\xi}\right)$ consists of two terms: one is due to the multipath with timing offset $e_{\xi}$, and the other is due to channel noise and interferences. If the channel statistics are known, the estimated channel autocorrelation $\mathbf{C}_{\mathbf{n}}\left(e_{\xi}\right)$ can be derived for any given $e_{\xi}$.

\section{AN MLE OF THE SymbOL TIMING ERROR}

This section derives the MLE of the timing offset based on the system model presented in Section II. According to (15), the negative log-likelihood function of the symbol timing error can be expressed as

$$
\Lambda\left(e_{\xi}\right)=K_{1}+\hat{\mathbf{h}}^{H} \mathbf{C}_{\mathbf{n}}^{-\mathbf{1}}\left(e_{\xi}\right) \hat{\mathbf{h}}
$$

where $K_{1}=N \ln \pi+\ln \left[\left|\operatorname{det}\left(\mathbf{C}_{\mathbf{n}}\left(e_{\xi}\right)\right)\right|\right]$. It can be shown that $\operatorname{det}\left(\mathbf{C}_{\mathbf{n}}\left(e_{\xi}\right)\right)$ is actually independent of $e_{\xi}$ (see Appendix I), thus $K_{1}$ is a constant with respect to $e_{\xi}$. The MLE of the timing offset $e_{\xi}$ is simply given by

$$
e_{\xi M L}=\arg \min _{e_{\xi}}\left[\hat{\mathbf{h}}^{H} \mathbf{C}_{\mathbf{n}}^{-\mathbf{1}}\left(e_{\xi}\right) \hat{\mathbf{h}}\right]
$$

Note that to obtain this MLE of the timing offset, we need the knowledge of the channel statistics at the receiver. Much has been done on the estimation of the OFDM channel statistics, see, e.g., [25], [26]. Thus it is not necessary to address this issue here and we simply assume that the channel statistics are known at the receiver.

One can see that the ML timing estimator as formulated in (19) may be too complex to be practical, as it involves the inversion of an $N \times N$ autocorrelation matrix. However, a closer examination of $\mathbf{C}_{\mathbf{n}}\left(e_{\xi}\right)$ helps us simplify this estimator so that it is more practical.

In the following sections, we will study the simplifications for the proposed MLE for integer precision as well as for realvalued precision. With these simplifications, we can obtain the 
ML solution much more efficiently for different implementation requirements.

\section{MLE FOR INTEGER PRECISION}

Many typical OFDM timing synchronization schemes provide an integer timing resolution, see, e.g., [27]. We shall thus first examine the proposed MLE (19) for an integer-valued precision in this section.

\section{A. Derivation of the MLE for Integer Precision}

To begin, we note that the dominant elements of the channel autocorrelation matrix $\mathbf{C}_{\mathbf{n}}(0)$ are only located in a $P \times P$ submatrix along the diagonal, where $P$ is the length of the PDP. Note also that $P$ is comparable to the guard interval $N_{c p}$ and is much smaller than the FFT size $N$. Considering the possible timing offset in the region $[-E, E]$, we choose a truncated $Q$-length vector of $\hat{\mathbf{h}}(14)$, where $Q=P+2 E$, to cover the entire channel PDP. This truncated version of $\hat{\mathbf{h}}$ is denoted as $\hat{\mathbf{h}}_{\mathbf{t c}}$. A corresponding autocorrelation submatrix $\mathbf{C}_{\mathbf{n}_{-\mathbf{t c}}}(0)$ is truncated from $\mathbf{C}_{\mathbf{n}}(0)$ (starting with the $(I, I)$-th element). Similarly to (15), the probability density function of $\hat{\mathbf{h}}_{\mathbf{t c}}\left(e_{\xi}\right)$ can be expressed as

$$
\begin{aligned}
p\left(\hat{\mathbf{h}}_{\mathbf{t c}}\left(e_{\xi}\right) ; e_{\xi}\right)= & \frac{1}{\pi^{Q}\left|\operatorname{det}\left(\mathbf{C}_{\mathbf{n} \_\mathbf{t c}}\left(e_{\xi}\right)\right)\right|} \\
& \times \exp \left[-\hat{\mathbf{h}}_{\mathbf{t c}}^{H}\left(e_{\xi}\right) \mathbf{C}_{\mathbf{n}_{-} \mathbf{t c}}^{-\mathbf{1}}\left(e_{\xi}\right) \hat{\mathbf{h}}_{\mathbf{t c}}\left(e_{\xi}\right)\right]
\end{aligned}
$$

Note that $\operatorname{det}\left(\mathbf{C}_{\mathbf{n}_{-} \text {tc }}\left(e_{\xi}\right)\right)$ is almost equal to $\operatorname{det}\left(\mathbf{C}_{\mathbf{n}}\left(e_{\xi}\right)\right)$ due to the truncation of the insignificant part of $\mathbf{C}_{\mathbf{n}}\left(e_{\xi}\right)$. Therefore, $\operatorname{det}\left(\mathbf{C}_{\mathbf{n}_{-} \mathbf{t c}}\left(e_{\xi}\right)\right)$ can be considered constant with respect to $e_{\xi}$, just like $\operatorname{det}\left(\mathbf{C}_{\mathbf{n}}\left(e_{\xi}\right)\right)$. Henceforth, an MLE based on (20) can be expressed as

$$
\hat{e}_{\xi M L}=\arg \min _{e_{\xi}}\left[\hat{\mathbf{h}}_{\mathbf{t c}}^{H} \mathbf{C}_{\mathbf{n}_{-} \mathbf{t c}}^{-\mathbf{1}}\left(e_{\xi}\right) \hat{\mathbf{h}}_{\mathbf{t c}}\right]
$$

Compared to (19), the dimension of the inverse matrix in (21) is much smaller.

Secondly, for an integer-valued timing offset $e_{\xi}$, it is easy to see that the channel autocorrelation matrix $\mathbf{C}_{\mathbf{n}}\left(e_{\xi}\right)$ (or $\mathbf{C}_{\mathbf{n}-\mathbf{t c}}\left(e_{\xi}\right)$ ) is simply an $e_{\xi}$ (integer) shift of the zero-offset channel autocorrelation matrix $\mathbf{C}_{\mathbf{n}}(0)$ (or $\mathbf{C}_{\mathbf{n}_{\mathbf{t}} \mathbf{t c}}(0)$ ). Its calculation is thus a trivial task. Moreover, if the channel autocorrelation matrix is diagonally dominant, the matrix inversion can be approximated by simply inverting its diagonal elements. Specifically, denote the diagonal elements of $\mathbf{C}_{\mathbf{n}}(0)$ by a vector $\left\{c_{1}, c_{2}, \ldots, c_{N}\right\}$, which consists of the channel energy (i.e., the channel PDP) and the additive noise (17). The MLE can be simplified as

$$
\begin{aligned}
\Lambda\left(e_{\xi}\right) & =\sum_{1 \leq k \leq Q} \frac{\left|\hat{h}_{t c, k}\right|^{2}}{c_{I-1+k+e_{\xi}}} \\
& =\sum_{1 \leq k \leq Q}\left|\hat{h}_{t c, k}\right|^{2} \cdot\left(\frac{1}{c_{I-1+k+e_{\xi}}}\right) \\
\hat{e}_{\xi M L} & =\arg \min _{e_{\xi}}\left[\Lambda\left(e_{\xi}\right)\right]
\end{aligned}
$$

Note that the channel PDP is located in the range $t \in[I+E, I+$ $E+P-1]$ of the vector $\left\{c_{t}\right\}$. The final expression (22) can be viewed as the correlation between the power of the estimated CIR $\left(\left|\hat{h}_{t c, k}\right|^{2}\right)$ and the reciprocal of the sum of the channel PDP and the additive noise $\left(1 / c_{k}\right)$. Such a correlation can be efficiently implemented using common digital signal processors.

\section{B. Analysis of the Proposed MLE for Integer Precision}

In this section, we analyze the proposed MLE in integer precision and compare its performance with those of other timing schemes based on the metric of correct estimation probability. We start with the conventional peak-finding method, [17], [19], and generalize it here as one of finding the position of the maximum amplitude in the estimated CIR $\left(\hat{\mathbf{h}}_{\mathbf{t c}}\right)$, i.e.,

$$
\hat{e}_{\xi P F}=\arg \max _{e_{\xi}}\left[\left|\hat{h}_{t c, e_{\xi}}\right|\right]
$$

To simplify the analysis, assume that the samples $\left\{\hat{h}_{t c, k}\right\}$ are independent Gaussian random variables with variance $\sigma_{k}^{2}$, and $\hat{h}_{t c, K}$ is the one with the largest variance $\sigma_{K}^{2}$ ( $K$ is then the peak position in the statistical sense, i.e., the correct timing position). The correct estimation probability is thus

$$
P_{P F 0}=P\left(\left|\hat{h}_{t c, K}\right|>\left|\hat{h}_{t c, k}\right|, \text { for } k \in[1 \ldots Q] \text { and } k \neq K\right)
$$

This probability can be calculated as, (see Appendix III)

$$
\begin{aligned}
P_{P F 0}= & 1-\frac{1}{\sigma_{K}^{2}} \sum_{k \neq K} \frac{1}{\frac{1}{\sigma_{K}^{2}}+\frac{1}{\sigma_{k}^{2}}} \\
& +\frac{1}{\sigma_{K}^{2}} \sum_{\substack{j \neq k \\
j \neq K, k \neq K}} \frac{1}{\frac{1}{\sigma_{K}^{2}}+\frac{1}{\sigma_{j}^{2}}+\frac{1}{\sigma_{k}^{2}}} \cdots \\
& (-1)^{Q-1} \frac{1}{\sigma_{K}^{2}} \frac{1}{\frac{1}{\sigma_{1}^{2}}+\frac{1}{\sigma_{2}^{2}}+\cdots+\frac{1}{\sigma_{Q}^{2}}}
\end{aligned}
$$

A simple upper bound for $P_{P F 0}$ is given by

$$
P_{P F 0}<P\left(\left|\hat{h}_{t c, K}\right|>\max _{k \neq K}\left|\hat{h}_{t c, k}\right|\right)=\frac{\sigma_{K}^{2}}{\sigma_{K}^{2}+\max _{k \neq K} \sigma_{k}^{2}}
$$

Next, we examine the proposed MLE given in (22). Again, we assume that $\hat{\mathbf{h}}_{\mathbf{t c}}$ has an autocorrelation $\mathbf{C}_{\mathbf{n}_{-} \mathbf{t c}}(\mathbf{0})$, and $\left\{\hat{h}_{t c, k}\right\}$ are independent Gaussian random variables with variance $\sigma_{k}^{2}$. The correct estimation probability for the MLE is thus

$$
P_{M L 0}=P\left(\Lambda(0)<\Lambda\left(e_{\xi}\right), \text { for any } e_{\xi} \neq 0\right)
$$

As shown in Appendix III, the probability of $P\left(\Lambda(0)<\Lambda\left(e_{\xi}\right)\right)$ is given by

$$
\begin{aligned}
P\left(\Lambda(0)<\Lambda\left(e_{\xi}\right)\right) & =\sum_{\substack{k=1 \\
w i t h \lambda_{k}<0}}^{Q} A_{k} \\
\text { with } \lambda_{k} & =\frac{1}{\sigma_{k}^{2}}-\frac{1}{\sigma_{k+e_{\xi}}^{2}}, \\
A_{k} & =\prod_{\substack{i=1 \\
i \neq k}}^{Q} \frac{\sigma_{k}^{2} \lambda_{k}}{\sigma_{k}^{2} \lambda_{k}-\sigma_{i}^{2} \lambda_{i}}
\end{aligned}
$$




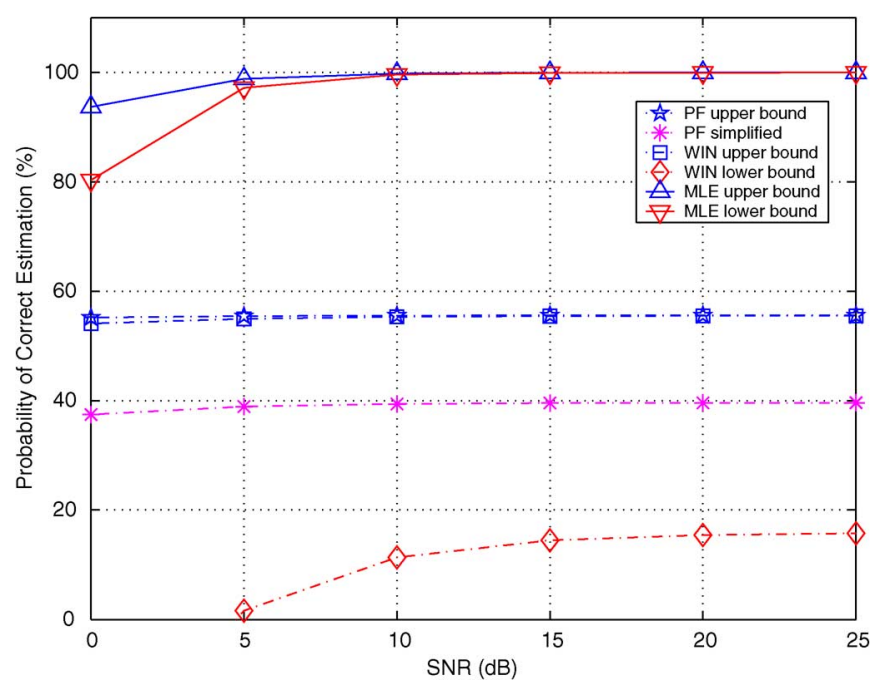

Fig. 1. Correct estimation probabilities for the peak-finding, the maximumenergy-windowing and the integer precision MLE method.

TABLE I

COST207 12-Multipath TyPiCAL URBAN (TU) ChanNEL Model

\begin{tabular}{|c|c|c|c|c|c|c|}
\hline Path Delay $(\mu \mathrm{s})$ & 0 & 0.1 & 0.3 & 0.5 & 0.8 & 1.1 \\
\hline Ave. Power $(\mathrm{dB})$ & -4.0 & -3.0 & 0 & -2.6 & -3.0 & -5.0 \\
\hline Path Delay $(\mu \mathrm{s})$ & 1.3 & 1.7 & 2.3 & 3.1 & 3.2 & 5.0 \\
\hline Ave. Power $(\mathrm{dB})$ & -7.0 & -5.0 & -6.5 & -8.6 & -11.0 & -10.0 \\
\hline
\end{tabular}

A lower bound for $P_{M L 0}$ can be obtained as

$$
\begin{aligned}
P_{M L 0} & =P\left(\bigcap_{e_{\xi}}\left(\Lambda(0)<\Lambda\left(e_{\xi}\right)\right)\right) \\
& =1-P\left(\bigcup_{e_{\xi}}\left(\Lambda(0) \geq \Lambda\left(e_{\xi}\right)\right)\right) \\
& >1-\sum_{e_{\xi}} P\left(\Lambda(0) \geq \Lambda\left(e_{\xi}\right)\right) \\
& =1-\sum_{e_{\xi}}\left(1-P\left(\Lambda(0)<\Lambda\left(e_{\xi}\right)\right)\right)
\end{aligned}
$$

and an upper bound is simply

$$
P_{M L 0}<\min _{e_{\xi}} P\left(\Lambda(0)<\Lambda\left(e_{\xi}\right)\right)
$$

Finally, we investigate another frequency-domain-based timing scheme which is designed to locate the position of the maximum CIR energy window [16], [21], [22]. The timing metric for this timing synchronizer can be expressed in general as

$$
\begin{aligned}
M\left(e_{\xi}\right) & =\sum_{k=I}^{I+Q-1}\left|\hat{h}_{k-e_{\xi}}\right|^{2} \\
\hat{e}_{\xi W I N} & =\arg \max _{e_{\xi}}\left[M\left(e_{\xi}\right)\right]
\end{aligned}
$$

where $I$ is the starting position of the energy window with zero timing offset and $Q$ is the window length. Assume similarly that $\left\{\hat{h}_{k}\right\}$ are independent Gaussian random variables with variance $\sigma_{k}^{2}$. Without loss of generality, we assume that the zero timing offset window has the largest CIR energy. The correct timing estimation probability can thus be expressed as

$$
P_{W I N 0}=P\left(M(0)>M\left(e_{\xi}\right), \text { for any } e_{\xi} \neq 0\right)
$$

Probability $P\left(M(0)>M\left(e_{\xi}\right)\right)$ can be computed as

$$
\begin{aligned}
P\left(M(0)>M\left(e_{\xi}\right)\right)= & \sum_{k=1}^{e_{\xi}} A_{k+},\left(\text { for } e_{\xi}>0\right) \\
\text { with } A_{k+}= & \prod_{\substack{i=1 \\
i \neq k}}^{e_{\xi}} \frac{\sigma_{I+Q-k}^{2}}{\sigma_{I+Q-k}^{2}-\sigma_{I+Q-i}^{2}} \\
& \times \prod_{i=1}^{e_{\xi}} \frac{\sigma_{I+Q-k}^{2}}{\sigma_{I+Q-k}^{2}+\sigma_{I-i}^{2}} \\
P\left(M(0)>M\left(e_{\xi}\right)\right)= & \sum_{k=1}^{-e_{\xi}} A_{k+},\left(\text { for } e_{\xi}<0\right) \\
\text { with } A_{k+}= & \prod_{\substack{i=1 \\
i \neq k}}^{-e_{\xi}} \frac{\sigma_{I+k-1}^{2}}{\sigma_{I+k-1}^{2}-\sigma_{I+i-1}^{2}} \\
& \times \prod_{i=1}^{-e_{\xi}} \frac{\sigma_{I+k-1}^{2}}{\sigma_{I+k-1}^{2}+\sigma_{I+Q+i-1}^{2}}
\end{aligned}
$$

Henceforth, a lower bound for $P_{W I N 0}$ can be derived as

$$
\begin{aligned}
P_{W I N 0} & =P\left(\bigcap_{e_{\xi}}\left(M(0)>M\left(e_{\xi}\right)\right)\right) \\
& =1-P\left(\bigcup_{e_{\xi}}\left(M(0) \leq M\left(e_{\xi}\right)\right)\right) \\
& >1-\sum_{e_{\xi}} P\left(M(0) \leq M\left(e_{\xi}\right)\right) \\
& =1-\sum_{e_{\xi}}\left(1-P\left(M(0)>M\left(e_{\xi}\right)\right)\right)
\end{aligned}
$$

and a simple upper bound for $P_{W I N O}$ is simply given by

$$
P_{W I N 0}<\min _{e_{\xi}} P\left(M(0)>M\left(e_{\xi}\right)\right)
$$

Employing the COST207 TU model (Table I), we calculated the approximate correct estimation probabilities for these timing synchronizers using the above formulas and showed the results in Fig. 1. For the peak-finding method, the 'PF upper bound' curve is obtained by using (27) with $Q=30$ and the 'PF simplified' curve is obtained using (26) with $Q=7$; for the integer precision MLE method, the curves are generated by using (29) with $Q=30$ and $\Lambda(\cdot)$ being evaluated in a timing offset region of $[-3,3]$; as for the maximum-energy-windowing method, curves are provided by (32) with $Q$ fine-tuned to 7 for the best performance, and $M(\cdot)$ evaluated in a timing offset region of $[-5,5]$. The timing offset region is set by assuming that a good early-stage coarse timing has been done and the probability of timing error within the region is close to 1 for tracking by this scheme. It can be observed from Fig. 1 that the maximum-energy-windowing method and the peak-finding 


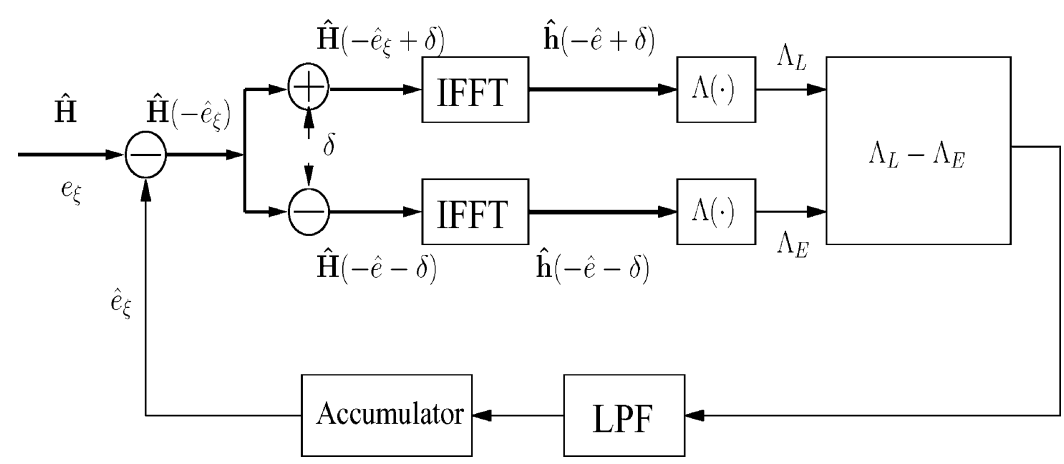

Fig. 2. The proposed PDP-based fine timing delay-locked loop.

method have comparable timing estimation capability, while the proposed MLE performs significantly better than those two other schemes.

\section{Real-VALUed PRECISION AND THE DLL StRUCTURE}

\section{A. Design of the DLL Structure for Real-Valued Precision $M L E$}

Real-valued precision fine timing has been used in many OFDM systems [8], [17], [20]. In the case of estimating the timing offset with a real-valued precision, it is computationally too complex to find the MLE through (19) and even with the simplified version (21). Searching for the MLE in a fractional timing grid is not a practical solution. Alternatively, the delay locked loop (DLL) in [17] offers a more practical solution. Assuming that the initial estimate of $e_{\xi}$ is within the convergence region, a recursive solution for the MLE is given by

$$
\hat{e}_{\xi}(i+1)=\hat{e}_{\xi}(i)-\mu \frac{d \Lambda\left(\hat{e}_{\xi}(i)\right)}{d e_{\xi}}
$$

Here, $\mu$ is a small positive number and the derivative in (37) can be approximated by

$$
\frac{d \Lambda\left(\hat{e}_{\xi}(i)\right)}{d e_{\xi}} \approx \frac{1}{2 \delta}\left[\Lambda\left(\hat{e}_{\xi}(i)+\delta\right)-\Lambda\left(\hat{e}_{\xi}(i)-\delta\right)\right]
$$

with $\delta$ denoting the advanced/retarded interval. This results in a recursive solution given by

$$
\begin{aligned}
\hat{e}_{\xi}(i+1)=\hat{e}_{\xi}(i)-k[ & {\left[\left(\hat{e}_{\xi}(i)+\delta\right)-\Lambda\left(\hat{e}_{\xi}(i)-\delta\right)\right] } \\
=\hat{e}_{\xi}(i)-k[ & \hat{\mathbf{h}}^{H} \mathbf{C}_{\mathbf{n}}^{-\mathbf{1}}\left(\hat{e}_{\xi}(i)+\delta\right) \hat{\mathbf{h}} \\
& \left.-\hat{\mathbf{h}}^{H} \mathbf{C}_{\mathbf{n}}^{-\mathbf{1}}\left(\hat{e}_{\xi}(i)-\delta\right) \hat{\mathbf{h}}\right]
\end{aligned}
$$

This is actually a first-order digital DLL with a loop coefficient $k=\mu / 2 \delta$.

Furthermore, it is much simpler to calculate $\hat{\mathbf{h}}\left(e_{\xi}\right)$ than to calculate $\mathbf{C}_{\mathbf{n}}\left(e_{\xi}\right)$ for an arbitrary timing offset $e_{\xi}$. More specifically, $\hat{\mathbf{h}}\left(e_{\xi}\right)$ can be obtained by introducing a phase adjustment in the frequency-domain as

$$
\begin{aligned}
\hat{\mathbf{h}}\left(e_{\xi}\right) & =\frac{1}{N} \mathbf{W}^{H} \hat{\mathbf{H}}\left(e_{\xi}\right) \\
& =\frac{1}{N} \mathbf{W}^{H} \mathbf{R}\left(e_{\xi}\right) \hat{\mathbf{H}}(0)
\end{aligned}
$$

where, $\mathbf{R}\left(e_{\xi}\right)$ is a diagonal matrix with elements $\left\{e^{j(2 \pi / N) n e_{\xi}}\right\}$. Thereafter, instead of using $\hat{\mathbf{h}}^{H} \mathbf{C}_{\mathbf{n}}^{-\mathbf{1}}\left(e_{\xi}\right) \hat{\mathbf{h}}$ in (19), we can use an alternative formula $\hat{\mathbf{h}}^{H}\left(-e_{\xi}\right) \mathbf{C}_{\mathbf{n}}^{-\mathbf{1}}(0) \hat{\mathbf{h}}\left(-e_{\xi}\right)$ to calculate the log-likelihood function (see Appendix II)

$$
\hat{\mathbf{h}}^{H}(0) \mathbf{C}_{\mathbf{n}}^{-\mathbf{1}}\left(e_{\xi}\right) \hat{\mathbf{h}}(0)=\hat{\mathbf{h}}^{H}\left(-e_{\xi}\right) \mathbf{C}_{\mathbf{n}}^{-\mathbf{1}}(0) \hat{\mathbf{h}}\left(-e_{\xi}\right)
$$

Similarly, we replace $\quad \hat{\mathbf{h}}_{\mathbf{t c}}^{H} \mathbf{C}_{\mathbf{n}-\mathbf{t c}}^{-\mathbf{1}}\left(e_{\xi}\right) \hat{\mathbf{h}}_{\mathbf{t c}} \quad$ by $\hat{\mathbf{h}}_{\mathbf{t c}}^{H}\left(-e_{\xi}\right) \mathbf{C}_{\mathbf{n} \_\mathbf{t c}}^{-1}(0) \hat{\mathbf{h}}_{\mathbf{t c}}\left(-e_{\xi}\right)$ to calculate the simplified log-likelihood function in (21).

Combining all those factors, we eventually obtain a DLL structure as

$$
\begin{aligned}
\hat{e}_{\xi}(i+1)= & \hat{e}_{\xi}(i)-k\left[\hat{\mathbf{h}}_{\mathbf{t c}}^{H}\left(-\hat{e}_{\xi}(i)-\delta\right) \mathbf{C}_{\mathbf{n} \_\mathbf{t c}}^{-\mathbf{1}}(0) \hat{\mathbf{h}}_{\mathbf{t c}}\left(-\hat{e}_{\xi}(i)-\delta\right)\right. \\
& \left.-\hat{\mathbf{h}}_{\mathbf{t c}}^{H}\left(-\hat{e}_{\xi}(i)+\delta\right) \mathbf{C}_{\mathbf{n}_{-\mathbf{t c}}}^{-\mathbf{1}}(0) \hat{\mathbf{h}}_{\mathbf{t c}}\left(-\hat{e}_{\xi}(i)+\delta\right)\right]
\end{aligned}
$$

Applying the diagonal simplification to the matrix $\mathbf{C}_{\mathbf{n}_{-} \mathbf{t c}}(0)$, similarly to the way that we obtained (22), we get

$$
\begin{aligned}
\hat{e}_{\xi}(i+1)=\hat{e}_{\xi}(i)-k & {\left[\sum_{1 \leq k \leq Q} \frac{\left|\hat{h}_{t c, k}\left(-e_{\xi}(i)-\delta\right)\right|^{2}}{c_{I-1+k}}\right.} \\
& \left.-\sum_{1 \leq k \leq Q} \frac{\left|\hat{h}_{t c, k}\left(-e_{\xi}(i)+\delta\right)\right|^{2}}{c_{I-1+k}}\right]
\end{aligned}
$$

where $c_{k}$ is the diagonal element of $\mathbf{C}_{\mathbf{n}}(0)$. This results in the PDP-based DLL structure, which is depicted in Fig. 2. In practice, the integer part of the estimated timing offset is usually adjusted in the time domain and the fractional part of the offset can be efficiently compensated by phase rotation in the frequency domain. (Some applications compensate the timing error by fine tuning the sampling clock.)

\section{B. S-Curve Analysis for the MLE DLL}

For a DLL, the discriminator characteristic (S-curve) is the average value of the discriminator output as a function of the timing error [28]. It is an important design factor to determine the loop tracking ability, i.e., the dynamic handling capability and the tracking error variance. In this section we will study the discriminator characteristic of the proposed DLL structure for the real-valued precision timing MLE. Considering the metric in the recursive solution (39), we denote

$$
M\left(\hat{e}_{\xi}(i)\right)=\hat{\mathbf{h}}^{H} \mathbf{C}_{\mathbf{n}}^{-\mathbf{1}}\left(\hat{e}_{\xi}(i)\right) \hat{\mathbf{h}}
$$


The S-curve of the DLL is

$$
\begin{aligned}
S\left(e_{\xi}\right) & =E\left[M\left(e_{\xi}+\delta\right)-M\left(e_{\xi}-\delta\right)\right] \\
& =E\left[M\left(e_{\xi}+\delta\right)\right]-E\left[M\left(e_{\xi}-\delta\right)\right]
\end{aligned}
$$

For the term $E\left[M\left(e_{\xi}\right)\right]$, we have

$$
\begin{aligned}
E\left[M\left(e_{\xi}\right)\right]= & E\left[\hat{\mathbf{h}}^{H} \mathbf{C}_{\mathbf{n}}^{-\mathbf{1}}\left(e_{\xi}\right) \hat{\mathbf{h}}\right]\{\operatorname{using}(14)\} \\
= & E\left[\left(\mathbf{h}^{H} \mathbf{B}^{H}(0)+\mathbf{V}^{H}\right) \mathbf{C}_{\mathbf{n}}^{-\mathbf{1}}\left(e_{\xi}\right)\left(\mathbf{B}^{H}(0) \mathbf{h}+\mathbf{V}\right)\right] \\
= & E\left[\mathbf{h}^{H} \mathbf{B}^{H}(0) \mathbf{C}_{\mathbf{n}}^{-\mathbf{1}}\left(e_{\xi}\right) \mathbf{B}^{H}(0) \mathbf{h}\right] \\
& +E\left[\mathbf{V}^{H} \mathbf{C}_{\mathbf{n}}^{-\mathbf{1}}\left(e_{\xi}\right) \mathbf{V}\right]
\end{aligned}
$$

where $\mathbf{B}\left(e_{\xi}\right)=(1 / N) \mathbf{W}^{H} \mathbf{M}\left(e_{\xi}\right)$ and $\mathbf{V}=$ $(1 / N) \mathbf{W}^{H} \mathbf{X}^{-1} \mathbf{Z}$. We further denote $\mathbf{B}^{H}(0) \mathbf{C}_{\mathbf{n}}^{-\mathbf{1}}\left(e_{\xi}\right) \mathbf{B}^{H}(0)$ as $\mathbf{Q}\left(e_{\xi}\right)$ and approximate $E\left[\mathbf{V}^{H} \mathbf{V}\right]$ as $(\beta / N \gamma) \mathbf{I}$, which leads to

$$
\begin{aligned}
E\left[M\left(e_{\xi}\right)\right] & \approx E\left[\sum_{l}\left|h_{l}\right|^{2} \mathbf{Q}\left(e_{\xi}\right)_{l l}\right]+\sum_{l} \frac{\beta}{N \gamma} \mathbf{C}_{\mathbf{n}}^{-\mathbf{1}}\left(e_{\xi}\right) l l \\
& =\sum_{l} \sigma_{l}^{2} \mathbf{Q}\left(e_{\xi}\right)_{l l}+\frac{\beta}{N \gamma} \operatorname{trace}\left[\mathbf{C}_{\mathbf{n}}^{-\mathbf{1}}\left(e_{\xi}\right)\right]
\end{aligned}
$$

Therefore, the S-curve of the DLL can be obtained as

$$
\begin{aligned}
S\left(e_{\xi}\right)= & E\left[M\left(e_{\xi}+\delta\right)\right]-E\left[M\left(e_{\xi}-\delta\right)\right] \\
\approx & \left.\left.\sum_{l} \sigma_{l}^{2}\left[\mathbf{Q}\left(e_{\xi}+\delta\right)\right)_{l l}-\mathbf{Q}\left(e_{\xi}-\delta\right)\right)_{l l}\right] \\
& +\frac{\beta}{N \gamma} \operatorname{trace}\left[\mathbf{C}_{\mathbf{n}}^{-\mathbf{1}}\left(e_{\xi}+\delta\right)-\mathbf{C}_{\mathbf{n}}^{-\mathbf{1}}\left(e_{\xi}-\delta\right)\right]
\end{aligned}
$$

As an example, the S-curve of the proposed ML timing estimator is calculated for the COST207 TU channel model with $\delta$ equal 0.5 and the result is shown in Fig. 3. It is seen from the figure that the S-curve of the ML timing estimator is a function of channel SNR. The shape of the S-curve is also dependent on the value of $\delta$ and the channel PDP. The derivative of the S-curve around the origin is considered the discriminator gain in the design of DLL, depicted in Fig. 2.

\section{Simulation Results}

In our simulation experiments, a BPSK-OFDM system was considered with $N=512$ subchannels and a bandwidth of 4.096 MHz operating in the $1.8 \mathrm{GHz}$ frequency band. The effective OFDM symbol duration $T_{u}$ was $125 \mu$ s and the sampling period $T$ was $0.244 \mu \mathrm{s}$. The guard interval consisted of a 52-sample cyclic prefix. Sixty-four pilot subchannels were equally spaced among the 512 subchannels to transmit the same strength BPSK pilot signals. Again the COST207 TU channel model was employed here for the simulation.

First, we investigate the integer-valued precision MLE scheme. Each multipath in the TU model experiences a time-varying fading. The proposed MLE synchronizer, the first-peak-finding (FPF) synchronizer and the maximum-energy-windowing (MEW) synchronizer are studied and compared using a time-varying fading channel. The simulation results are shown in Fig. 4 in terms of the empirical probability mass function (PMF), i.e., the timing offset histogram for

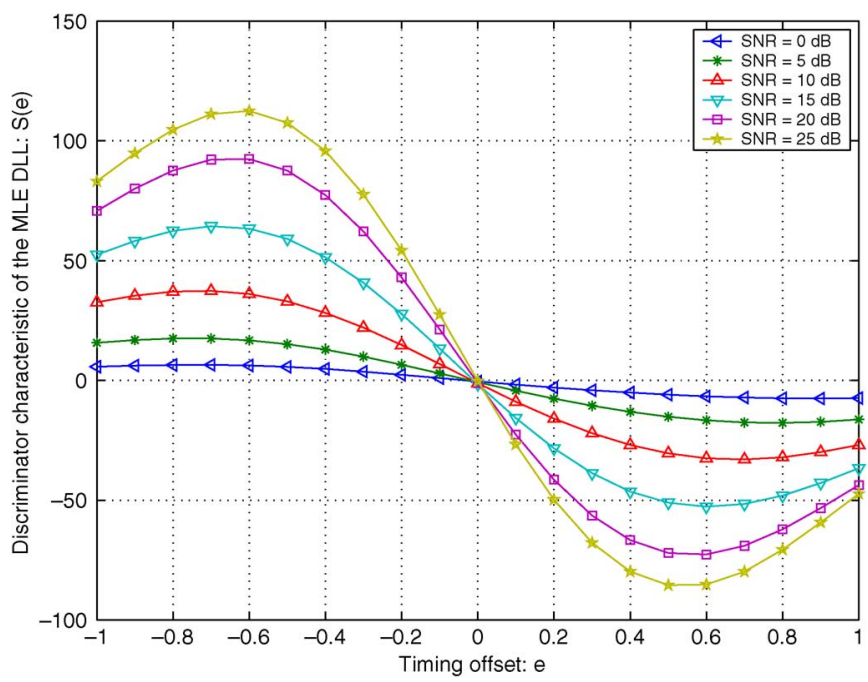

Fig. 3. S-curve of the MLE DLL using the COST207 TU model. $(\delta=0.5)$.

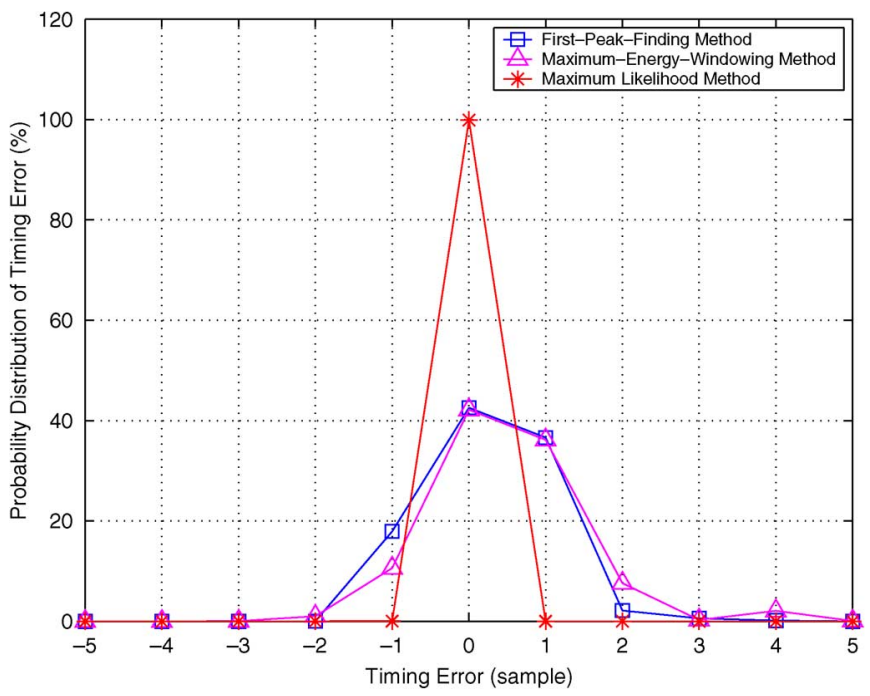

Fig. 4. Histograms for the first-peak-finding, maximum-energy-windowing and the integer precision MLE method. SNR $=20 \mathrm{~dB}$, with the TU model.

each synchronizer. The FPF scheme studied here is a modified peak-finding scheme, which applies a threshold to combat the noise effect and to find the first peak position in the estimated CIR. For the MEW scheme, the window length was fine-tuned to 7 to obtain the best performance under the specific channel. It is easily seen from the histogram that, at $\mathrm{SNR}=20 \mathrm{~dB}$, the proposed MLE synchronizer (error variance : 0.0026) performs much better than both the FPF synchronizer (error variance : 0.7115 ) and the MEW synchronizer (error variance : 1.1918).

Next, to examine the proposed MLE scheme for real-valued precision, the DLL structure proposed in Section V was simulated using the TU channel model with a $85 \mathrm{~Hz}$ Doppler spread. The advanced/retarded interval $\delta$ was set to be 0.5 , such that the two estimated CIRs would have a unit-one timing difference and can be simply obtained via one IFFT processing. The mean-square tracking performance (measured by the tracking error variance) of the proposed timing structure was investigated and compared with those applying the FPF and MEW timing schemes. The simulation results are presented in Fig. 5. 


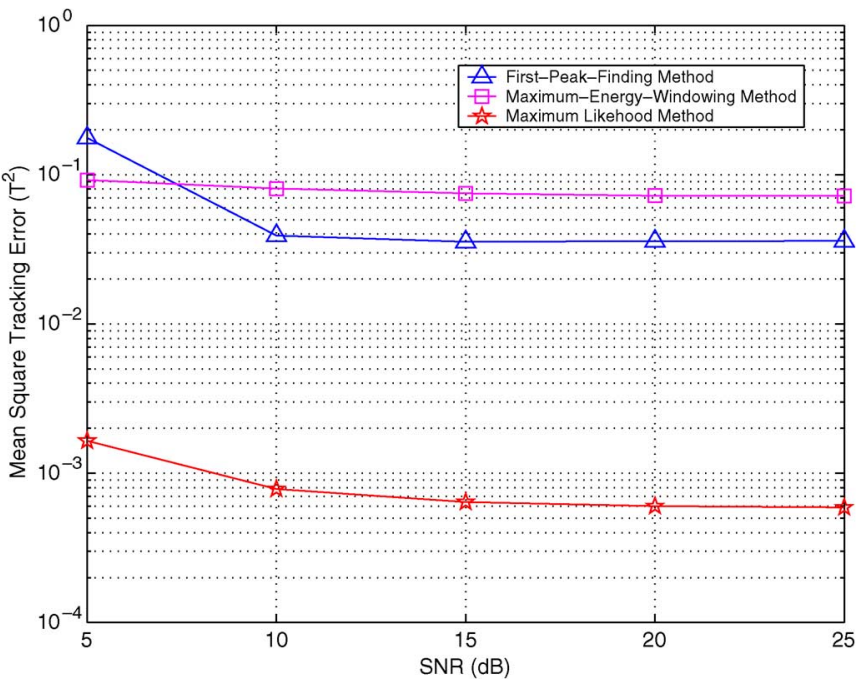

Fig. 5. Mean-square tracking error performance comparison of the first-peakfinding, the maximum-energy-windowing and the proposed scheme under the TU model $\left(N_{p}=64, B_{L}=0.02\right)$.

In all these DLL settings, first-order loop filters are adjusted according to their individual discriminator gains (the derivative of the S-curve) to have the same loop bandwidth $\left(B_{L}=0.02\right)$ for a fair comparison (Systems with the same loop bandwidth have comparable acquisition time [29]). From Fig. 5, it is easily seen that the FPF and the MEW methods have comparable performance and the MEW method exhibits better noise resistance than FPF does at low SNRs. Compared to those two schemes, our proposed ML timing scheme has much lower tracking error in the entire SNR region, yielding a nearly two orders of magnitude less mean-square error performance.

To further evaluate this timing performance gain for OFDM systems, we performed a system-level bit-error-rate (BER) simulation. As an example, we chose OFDM systems with a two-dimensional channel estimation here, i.e., the channel estimation is actually split into the frequency-direction and time-direction estimation. The frequency-direction channel estimation is carried out by using either the pilot-based MMSE [12] or the MLE [24]. The time-direction channel estimation then follows by employing an MMSE-based temporal interpolation. The temporal interpolation is done by inserting one pilot symbol in every eight OFDM symbols. Channel statistics and SNR are assumed known at the receiver to employ the MMSE channel estimates as well as the proposed ML fine timing estimation. Fig. 6 shows the results of these simulations. It is well known that the (frequency-direction) MMSE channel estimate is sensitive to the timing offset [8], [12] and our results also validate this point here. With perfect timing, the system using MMSE channel estimates provides a performance improvement of about $1.5 \mathrm{~dB}$ over that using ML40 channel estimate (the number of taps used in the ML/LS channel estimate [24] is 40). Fig. 6 also shows the system performance for different timing synchronizers. For the system using MMSE channel estimates, with the proposed ML fine timing scheme, it experiences a little performance loss compared to that with perfect timing. In contrast, the FPF and

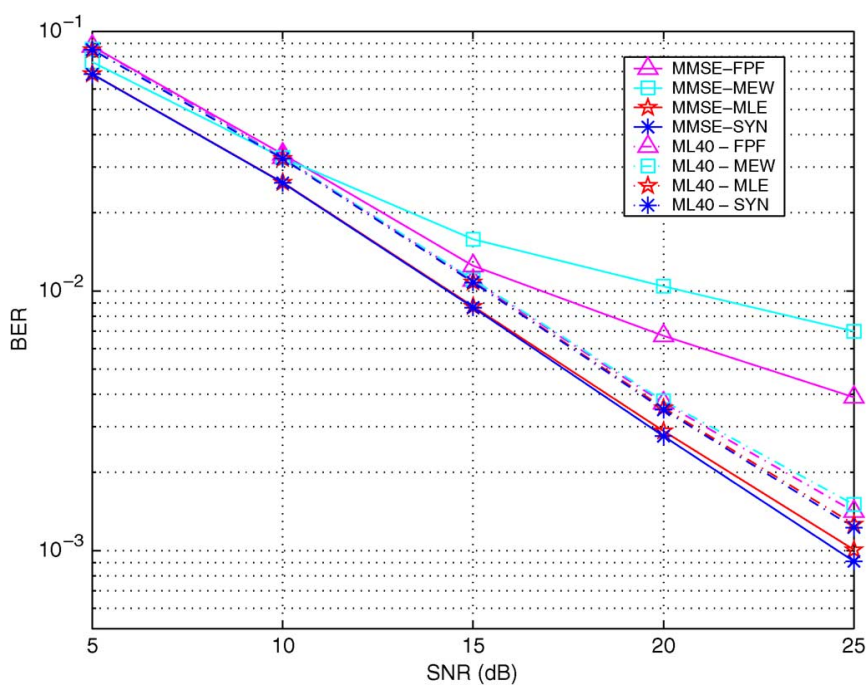

Fig. 6. BER performance comparison of different 2D channel estimates for different timing methods under the TU model.

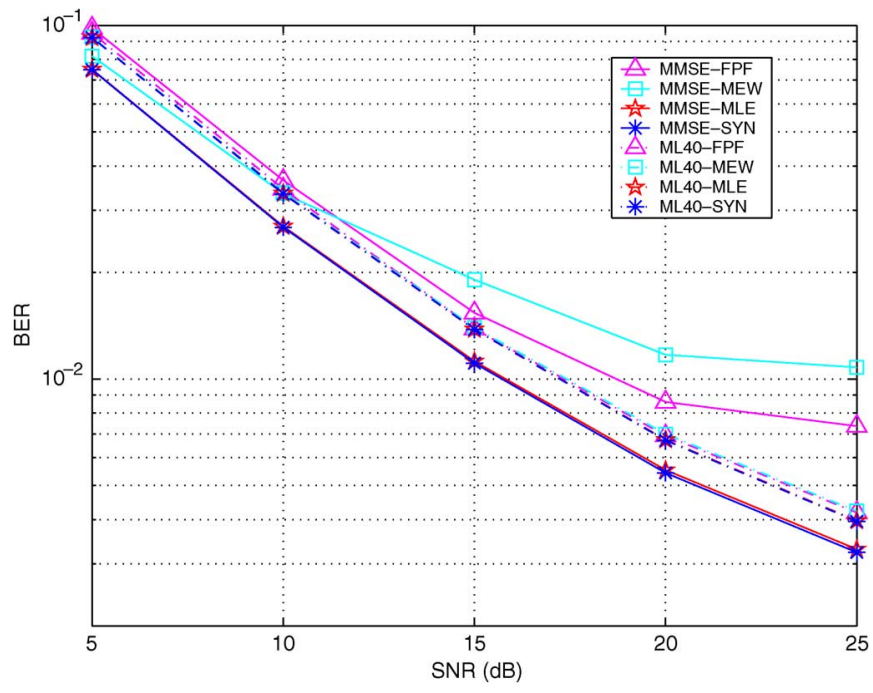

Fig. 7. BER performance comparison of different $2 \mathrm{D}$ channel estimates for different timing methods under the TU model with shortened CP.

MEW methods result in significant system performance loss, especially at high SNR. Similar simulations are provided to evaluate the system performance using ML channel estimate. The results show that ML channel estimates are not very sensitive to the timing error. In essence, with the aid of the proposed ML timing method, an MMSE-based system can retain its performance and outperform an ML-based system by $1.5 \mathrm{~dB}$ in BER.

Additional simulation experiments were carried out to examine ISI effects. In those experiments, we introduced an ISI effect on the OFDM system by shortening the cyclic prefix to 10 OFDM samples. Because the length of the channel delay profile is now longer than that of the cyclic prefix, there is energy leakage from adjacent OFDM symbols to the present one. Simulation results for different settings are shown in Fig. 7. Compared with Fig. 6, it is easily seen that the BER performance of both MMSE-based and ML-based systems are degraded due to the ISI effect. The performance difference between MMSE- 
based and ML-based systems under perfect timing situation has increased to about $2 \mathrm{~dB}$ at SNR around $20 \mathrm{~dB}$. Again, our ML timing scheme can be used to retain good performance of an MMSE-based system in the presence of ISI and provides a significant system performance gain over other schemes.

\section{CONCLUSION}

This paper has proposed an ML fine timing synchronization scheme for OFDM systems. The proposed scheme utilizes channel statistical information to improve the timing performance. This scheme can be simplified as an integer-valued timing synchronizer or, by employing a DLL structure, implemented with a real-valued timing precision. Performance of the proposed scheme has been evaluated through both analysis and simulation. Employing a COST207 TU wireless channel model, the proposed scheme is shown to be significantly better than the conventional schemes and achieves an improvement of nearly two orders of magnitude in terms of tracking error variance. Because the proposed scheme can reduce the timing jitter and provide an accurate timing match of channel statistics to the MMSE channel estimator, it helps to retain the optimum performance of the MMSE channel estimate and result in a system performance gain over those conventional systems applying the ML channel estimate.

\section{APPENDIX I}

\section{$\operatorname{det}\left[\mathbf{C}_{\mathbf{n}}\left(e_{\xi}\right)\right]$ IS INDEPENDENT OF $e_{\xi}$}

First, let us investigate $\mathbf{M}\left(e_{\xi 1}\right)^{H} \mathbf{M}\left(e_{\xi 2}\right)$, where $\mathbf{M}\left(e_{\xi}\right)_{N \times L}=\left[e^{j 2 \pi n\left(e_{\xi}-\tau_{l} / T\right) / N}\right]_{N \times L}$. The $(i, j)$ th element of $\mathbf{M}\left(e_{\xi 1}\right)^{H} \mathbf{M}\left(e_{\xi 2}\right)$ turns to be

$$
\mathbf{M}_{(i)}\left(e_{\xi 1}\right)^{H} \mathbf{M}_{(j)}\left(e_{\xi 2}\right)=\sum_{n=-N / 2}^{N / 2-1} e^{j \frac{2 \pi n\left(e_{\xi 2}-e_{\xi 1}+\tau_{i} / T-\tau_{j} / T\right)}{N}}
$$

Since (49) is only dependent on $\left(e_{\xi 2}-e_{\xi 1}\right)$, we can simply denote $\mathbf{M}\left(e_{\xi 1}\right)^{H} \mathbf{M}\left(e_{\xi 2}\right)$ as $\mathbf{F}\left(e_{\xi 2}-e_{\xi 1}\right)$.

Then study $\operatorname{det}\left[\mathbf{C}_{\mathbf{n}}\left(e_{\xi}\right)\right]$, according to (17), we have

$$
\begin{aligned}
\operatorname{det} & {\left[\mathbf{C}_{\mathbf{n}}\left(e_{\xi}\right)\right] } \\
& =\operatorname{det}\left[\frac{\beta}{N \gamma} \mathbf{I}+\frac{1}{N^{2}} \mathbf{W}^{H} \mathbf{M}\left(e_{\xi}\right) \mathbf{D}_{L} \mathbf{M}\left(e_{\xi}\right)^{H} \mathbf{W}\right] \\
& =\left(\frac{\beta}{N \gamma}\right)^{N} \operatorname{det}\left[\mathbf{I}+\frac{\gamma}{\beta N} \mathbf{W}^{H} \mathbf{M}\left(e_{\xi}\right) \mathbf{D}_{L} \mathbf{M}\left(e_{\xi}\right)^{H} \mathbf{W}\right] \\
& =\left(\frac{\beta}{N \gamma}\right)^{N} \operatorname{det}\left[\mathbf{I}+\frac{\gamma}{\beta N} \mathbf{D}_{L} \mathbf{M}\left(e_{\xi}\right)^{H} \mathbf{W} \mathbf{W}^{H} \mathbf{M}\left(e_{\xi}\right)\right] \\
& =\left(\frac{\beta}{N \gamma}\right)^{N} \operatorname{det}\left[\mathbf{I}+\frac{\gamma}{\beta} \mathbf{D}_{L} \mathbf{F}(0)\right]
\end{aligned}
$$

and this actually proves $\operatorname{det}\left[\mathbf{C}_{\mathbf{n}}\left(e_{\xi}\right)\right]$ is independent of $e_{\xi}$.

$$
\text { APPENDIX II }
$$

PRoOF: $\hat{\mathbf{h}}^{H}(0) \mathbf{C}_{\mathbf{n}}^{-\mathbf{1}}\left(e_{\xi}\right) \hat{\mathbf{h}}(0)=\hat{\mathbf{h}}^{H}\left(-e_{\xi}\right) \mathbf{C}_{\mathbf{n}}^{-\mathbf{1}}(0) \hat{\mathbf{h}}\left(-e_{\xi}\right)$

From the left-hand side of the equation, by using (14), (17) and (40), we have

$$
\hat{\mathbf{h}}^{H}(0) \mathbf{C}_{\mathbf{n}}^{-\mathbf{1}}\left(e_{\xi}\right) \hat{\mathbf{h}}(0)
$$

$$
\begin{aligned}
& =(\underbrace{\frac{1}{N} \mathbf{W}^{H} \mathbf{M}(0) \mathbf{h}}_{\mathrm{I}}+\underbrace{\frac{1}{N} \mathbf{W}^{H} \mathbf{X}^{-1} \mathbf{N}}_{\mathrm{II}})^{H} \\
& \times(\underbrace{\left.\frac{1}{N^{2}} \mathbf{W}^{H} \mathbf{M}\left(e_{\xi}\right) \mathbf{D}_{L} \mathbf{M}\left(e_{\xi}\right)^{H} \mathbf{W}+\frac{\beta}{N \gamma} \mathbf{I}\right)^{-1}}_{\mathrm{I}^{\prime}} \\
& \times(\underbrace{\frac{1}{N} \mathbf{W}^{H} \mathbf{M}(0) \mathbf{h}}_{\mathrm{II}^{\prime}}+\underbrace{\frac{1}{N} \mathbf{W}^{H} \mathbf{X}^{-1} \mathbf{N}})
\end{aligned}
$$

There are four terms coming out from $\mathrm{I} \times \mathrm{I}^{\prime}, \mathrm{I} \times \mathrm{II}^{\prime}, \mathrm{II} \times \mathrm{I}^{\prime}$ and $\mathrm{II} \times \mathrm{II}^{\prime}$ respectively. They can be derived individually as

$$
\begin{aligned}
\mathrm{I} \times \mathrm{I}^{\prime}= & \frac{\gamma}{\beta} \mathbf{h}^{H} \mathbf{F}(0) \mathbf{h}-\frac{N \gamma}{\beta} \mathbf{h}^{H} \mathbf{F}\left(e_{\xi}\right) \\
& \times\left(\frac{N \beta}{\gamma} \mathbf{D}_{L}^{-1}+\mathbf{F}(0)\right)^{-1} \mathbf{F}\left(-e_{\xi}\right) \mathbf{h} \\
\mathrm{I} \times \mathrm{II}^{\prime}= & \frac{\gamma}{\beta} \mathbf{h}^{H} \mathbf{M}^{H}(0) \mathbf{X}^{-1} \mathbf{N}-\frac{N \gamma}{\beta} \mathbf{h}^{H} \mathbf{F}\left(e_{\xi}\right) \\
& \times\left(\frac{N \beta}{\gamma} \mathbf{D}_{L}^{-1}+\mathbf{F}(0)\right)^{-1} \mathbf{M}^{H}\left(e_{\xi}\right) \mathbf{X}^{-1} \mathbf{N} \\
\mathrm{II} \times \mathrm{I}^{\prime}= & \frac{\gamma}{\beta} \mathbf{N}^{H}\left(\mathbf{X}^{-1}\right)^{H} \mathbf{M}(0) \mathbf{h}-\frac{N \gamma}{\beta} \mathbf{N}^{H}\left(\mathbf{X}^{-1}\right)^{H} \\
& \times \mathbf{M}^{-}\left(e_{\xi}\right)\left(\frac{N \beta}{\gamma} \mathbf{D}_{L}^{-1}+\mathbf{F}(0)\right)^{-1} \mathbf{F}\left(-e_{\xi}\right) \mathbf{h} \\
\mathrm{II} \times \mathrm{II}^{\prime}= & \frac{\gamma}{\beta} \mathbf{N}^{H}\left(\mathbf{X}^{-1}\right)^{H} \mathbf{X}^{-1} \mathbf{N}-\frac{N \gamma}{\beta} \mathbf{N}^{H}\left(\mathbf{X}^{-1}\right)^{H} \\
& \times \mathbf{M}^{-1}\left(e_{\xi}\right)\left(\frac{N \beta}{\gamma} \mathbf{D}_{L}^{-1}+\mathbf{F}(0)\right)^{-1} \mathbf{M}^{H}\left(e_{\xi}\right) \mathbf{X}^{-1} \mathbf{N}
\end{aligned}
$$

For the right-hand side of the equation, similarly we have

$$
\begin{aligned}
& \hat{\mathbf{h}}^{H}\left(-e_{\xi}\right) \mathbf{C}_{\mathbf{n}}^{-\mathbf{1}}(0) \hat{\mathbf{h}}\left(-e_{\xi}\right) \\
&=(\underbrace{\frac{1}{N} \mathbf{W}^{H} \mathbf{M}\left(-e_{\xi}\right) \mathbf{h}}_{\mathrm{I}}+\underbrace{\frac{1}{N} \mathbf{W}^{H} \mathbf{R}\left(-e_{\xi}\right) \mathbf{X}^{-1} \mathbf{N}}_{\mathrm{II}})^{H} \\
& \times(\underbrace{\left.\frac{1}{N^{2}} \mathbf{W}^{H} \mathbf{M}(0) \mathbf{D}_{L} \mathbf{M}(0)^{H} \mathbf{W}+\frac{\beta}{N \gamma} \mathbf{I}\right)^{-1}}_{\mathrm{I}^{\prime}} \\
& \times(\mathbf{W}_{\mathrm{II}}^{\frac{1}{N} \mathbf{W}^{H} \mathbf{M}\left(-e_{\xi}\right) \mathbf{h}}+\underbrace{\frac{1}{N} \mathbf{W}^{H} \mathbf{R}\left(-e_{\xi}\right) \mathbf{X}^{-1} \mathbf{N}})
\end{aligned}
$$

By using the fact $\mathbf{M}\left(e_{\xi 1}\right)=\mathbf{R}\left(e_{\xi 1}-e_{\xi 2}\right) \mathbf{M}\left(e_{\xi 2}\right)$, it can be easily shown that the four terms obtained from (56), i.e., $\mathrm{I} \times \mathrm{I}^{\prime}$, $\mathrm{I} \times \mathrm{II}^{\prime}, \mathrm{II} \times \mathrm{I}^{\prime}$ and $\mathrm{II} \times \mathrm{II}^{\prime}$, are equal to the four terms derived above from (51), such that we get the proof.

\section{APPENDIX III}

\section{The AnAlysis of THE Probabilities IN SECTION IV}

We first analyze the correct estimation probability of the generalized peak-finding scheme. Since the $\left\{\hat{h}_{i, k}\right\}$ are 
independently Gaussian distributed with variance $\sigma_{k}^{2}$, the $\left.\left\{z_{k}=\mid \hat{h}_{(} i, k\right) \mid\right\}$ will have a jointly distribution function as

$$
p_{z_{1} z_{2} \ldots z_{Q}}\left(z_{1}, z_{2}, \ldots, z_{Q}\right)=\prod_{n=1}^{Q}\left(z_{n} / \sigma_{n}^{2}\right) \exp \left(-z_{n}^{2} / 2 \sigma_{n}^{2}\right)
$$

The correct estimation probability (25) can be computed as

$$
\begin{aligned}
P_{P F 0}= & P\left(z_{K}>z_{k}, \text { for } k \in[1 \ldots Q] \text { and } k \neq K\right) \\
= & \int_{0}^{\infty} \frac{z_{K}}{\sigma_{K}^{2}} e^{-\frac{z_{K}^{2}}{2 \sigma_{K}^{2}}}\left(1-e^{-\frac{z_{K}^{2}}{2 \sigma_{1}^{2}}}\right) \ldots\left(1-e^{-\frac{z_{K}^{2}}{2 \sigma_{Q}^{2}}}\right) d z_{K} \\
= & 1-\frac{1}{\sigma_{K}^{2}} \sum_{k \neq K} \frac{1}{\frac{1}{\sigma_{K}^{2}}+\frac{1}{\sigma_{k}^{2}}} \\
& +\frac{1}{\sigma_{K}^{2}} \sum_{\substack{j \neq k \\
j \neq K, k \neq K}} \frac{1}{\frac{1}{\sigma_{K}^{2}}+\frac{1}{\sigma_{j}^{2}}+\frac{1}{\sigma_{k}^{2}}} \ldots \\
& (-1)^{Q-1} \frac{1}{\sigma_{K}^{2}} \frac{1}{\frac{1}{\sigma_{1}^{2}}+\frac{1}{\sigma_{2}^{2}}+\cdots+\frac{1}{\sigma_{Q}^{2}}}
\end{aligned}
$$

Next, we derive the probability of $P\left(\Lambda(0)<\Lambda\left(e_{\xi}\right)\right)$ in (29)

$$
\begin{aligned}
P(\Lambda(0) & \left.<\Lambda\left(e_{\xi}\right)\right) \\
= & P\left(\sum_{k=1}^{Q} \frac{\left|\hat{h}_{i, k}\right|^{2}}{\sigma_{k}^{2}}<\sum_{k=1}^{Q} \frac{\left|\hat{h}_{i, k}\right|^{2}}{\sigma_{k+e_{\xi}}^{2}}\right) \\
= & P\left(\sum_{k=1}^{Q}\left|\hat{h}_{i, k}\right|^{2}\left(\frac{1}{\sigma_{k}^{2}}-\frac{1}{\sigma_{k+e_{\xi}}^{2}}\right)<0\right) \\
= & \left\{\operatorname{denote} \lambda_{k}=\frac{1}{\sigma_{k}^{2}}-\frac{1}{\sigma_{k+e_{\xi}}^{2}}\right\} \\
= & P\left(\sum_{k=1}^{Q}\left|\hat{h}_{i, k}\right|^{2} \lambda_{k}<0\right) \\
= & P(\beta<0)
\end{aligned}
$$

here, $\beta=\sum_{k=1}^{Q}\left|\hat{h}_{i, k}\right|^{2} \lambda_{k}$. Since $\left\{\hat{h}_{i, k}\right\}$ are independently Gaussian distributed with variance $\sigma_{k}^{2}$, the characteristic function of $\beta$ is given by

$$
\begin{aligned}
\Phi_{\beta}(\omega) & =\prod_{k=1}^{Q} \frac{1}{1-j \omega \sigma_{k}^{2} \lambda_{k}} \\
& =\sum_{k=1}^{Q} A_{k} \frac{1}{1-j \omega \sigma_{k}^{2} \lambda_{k}} \\
\text { with } A_{k} & =\prod_{\substack{i=1 \\
i \neq k}}^{Q} \frac{\sigma_{k}^{2} \lambda_{k}}{\sigma_{k}^{2} \lambda_{k}-\sigma_{i}^{2} \lambda_{i}}
\end{aligned}
$$

The distribution of $\beta$ is thus

$$
P(\beta)=\left\{\begin{array}{l}
\sum_{k=1, k:\left(\lambda_{k}>0\right)}^{Q} \frac{A_{k}}{\sigma_{k}^{2} \lambda_{k}} e^{-\frac{\beta}{\sigma_{k}^{2} \lambda_{k}}}: \quad \beta>0 \\
\sum_{k=1, k:\left(\lambda_{k}<0\right)}^{Q} \frac{A_{k}}{\sigma_{k}^{2} \lambda_{k}} e^{-\frac{\beta}{\sigma_{k}^{2} \lambda_{k}}}: \quad \beta<0
\end{array}\right.
$$

which leads to equation (29) as

$$
P(\beta<0)=\sum_{\substack{k=1 \\ k:\left(\lambda_{k}<0\right)}}^{Q} A_{k}
$$

\section{REFERENCES}

[1] W. Y. Zou and Y. Wu, "COFDM: An overview," IEEE Trans. Broadcasting, vol. 41, no. 1, pp. 1-8, Mar. 1995.

[2] L. Hanzo, M. Munster, B. J. Choi, and T. Keller, OFDM and MC-CDMA for Broadband Multi-User Communications, WLANs and Broadcasting. Piscataway, NJ: IEEE Press, 2003.

[3] C. R. N. Athaudage, "BER sensitivity of OFDM systems to time synchronization error," in Proc. 8th IEEE Int. Conf. Communication Systems, Nov. 2002, pp. 42-46.

[4] X. Wang, T. T. Tjhuang, Y. Wu, and B. Caron, "SER performance evaluation and optimization of OFDM system with residual frequency and timing offset from imperfect synchronization," IEEE Trans. Broadcasting, vol. 49, no. 2, pp. 170-177, June 2003.

[5] T. Pollet, M. V. Bladel, and M. Moeneclaey, "BER sensitivity of OFDM systems to carrier frequency offset and Wiener phase noise," IEEE Trans. Communications, vol. 43, no. 2/3/4, pp. 191-193, Feb./Mar./Apr. 1995.

[6] M. Speth, S. Fechtel, G. Fock, and H. Meyr, "Optimum receiver design for wireless broad-band systems using OFDM-Part I," IEEE Trans. Communications, vol. 47, no. 11, pp. 1668-1677, Nov. 1999.

[7] Y. Mostofi and D. C. Cox, "Mathematical analysis of the impact of timing synchronization errors on the performance of an OFDM system," IEEE Trans. Communications, vol. 54, no. 2, pp. 226-230, Feb. 2006.

[8] M.-H. Hsieh and C.-H. Wei, "Channel estimation for OFDM systems based on comb-type pilot arrangement in frequency selective fading channels," IEEE Trans. Consumer Electronics, vol. 44, no. 1, pp. 217-225, Feb. 1998.

[9] Y. Mostofi, D. C. Cox, and A. Bahai, "Effect of frame synchronization errors on pilot-aided channel estimation in OFDM: Analysis and solution," in Proc. 5th International Symposium on Wireless Personal Multimedia Communications, Oct. 2002, pp. 1309-1313.

[10] Y. Mostofi and D. C. Cox, "Analysis of the effect of timing synchronization errors on pilot-aided OFDM systems," in Proc. Thirty-Seventh Asilomar Conference on Signals, Systems and Computers, 2003, Nov. 2003, vol. 1, pp. 9-12.

[11] Y. Mostofi and D. C. Cox, "Timing synchronization in high mobility OFDM systems," in Proc. IEEE International Conference on Communications, June 2004, pp. 2402-2406.

[12] C. R. N. Athaudage and A. D. S. Jayalath, "Enhanced MMSE channel estimation using timing error statistics for wireless OFDM systems," IEEE Trans. Broadcasting, vol. 50, no. 4, pp. 369-376, Dec. 2004.

[13] B. Yang, K. B. Letaief, R. S. Cheng, and Z. Cao, "Channel estimation for OFDM transmission in multipath fading channels based on parametric channel modeling," IEEE Trans. Communications, vol. 49, no. 3, pp. 467-479, July 2001.

[14] O. Simeone, Y. Bar-Ness, and U. Spagnolini, "Pilot-based channel estimation for OFDM systems by tracking the delay-subspace," IEEE Trans. Wireless Communications, vol. 3, no. 1, pp. 315-325, Jan. 2004.

[15] M. R. Raghavendra and K. Giridhar, "Improving channel estimation in OFDM Systems for sparse multipath channels," IEEE Signal Processing Letters, vol. 12, no. 1, pp. 52-55, Jan. 2005.

[16] M. Speth, S. Fechtel, G. Fock, and H. Meyr, "Optimum receiver design for OFDM-Based broadband transmission-Part II. A case study," IEEE Trans. Communications, vol. 49, no. 4, pp. 571-578, Apr. 2001.

[17] B. Yang, K. B. Letaief, R. S. Cheng, and Z. Cao, "Timing recovery for OFDM transmission," IEEE J. Selected Areas in Communications, vol. 18 , no. 11 , pp. 285-289, Nov. 2000.

[18] K. Yip, Y. Wu, and T. Ng, "Timing-synchronization analysis for IEEE 802.11a wireless LANs in frequency-nonselective Rician fading environments," IEEE Trans. Wireless Communications, vol. 3, no. 2, pp. 387-394, Mar. 2004.

[19] N. Chen, M. Tanaka, and R. Heaton, "OFDM timing synchronization under multi-path channels," in Proc. IEEE VTC, Apr. 2003, vol. 1, pp. $378-382$. 
[20] J. J. Kim, Y. J. Ryu, H. S. Oh, and D. S. Han, "Frame selection algorithm with adaptive FFT input for OFDM systems," in Proc. IEEE ICC, Apr. 2002, vol. 1, pp. 187-191.

[21] K. Wang, M. Faulkner, J. Singh, and I. Tolochko, "Timing synchronization for 802.11a WLANs under multipath channels," in Proc. ATNAC, Melbourne, Dec. 2003.

[22] H. Minn, V. K. Bhargava, and K. B. Letaief, "A robust timing and frequency synchronization for OFDM systems," IEEE Trans. Wireless Communications, vol. 2, no. 4, pp. 822-839, July 2003.

[23] H. Zhou and Y. F. Huang, "A maximum likelihood fine timing estimation for wireless OFDM systems," in Proc. 2005 IEEE 6th workshop on Signal Processing Advances in Wireless Communications, June 2005, pp. 705-709.

[24] J. J. van de Beek, O. Edfors, M. Sandell, S. K. Wilson, and P. O. Borjesson, "On channel estimation in OFDM systems," in Proc. IEEE VTC 1995, July 1995, pp. 815-819.

[25] H. Arslan and T. Yucek, "Estimation of frequency selectivity for OFDM based new generation wireless communication systems," in Proc. 2003 World Wireless Congress, San Francisco, May 2003.

[26] H. Schober and F. Jondral, "Delay spread estimation for OFDM based mobile communication systems," in Proc. European Wireless Conference, Florence, Italy, Feb. 2002, pp. 625-628.

[27] T. Keller, L. Piazzo, P. Mandarini, and L. Hanzo, "Orthogonal frequency division multiplex synchronization techniques for frequencyselective fading channels," IEEE J. Selected Areas in Communications, vol. 19, no. 6, pp. 999-1008, June 2001.

[28] J. C. Whitaker, The Electronics Handbook. Boca Raton, FL: CRC Press, 1996.

[29] U. Mengali and A. N. D’Andrea, Synchronization Techniques for Digital Receivers. New York: Plenum Press, 1997.

[30] A. I. Bo, G. E. Jian-Hua, and W. Yong, "Symbol synchronization technique in COFDM systems," IEEE Trans. Broadcasting, vol. 50, no. 1, pp. 56-62, March 2004

[31] B. Ai, Z.-X. Yang, C.-Y. Pan, J.-H. Ge, Y. Wang, and Z. Lu, "On the synchronization techniques for wireless OFDM systems," IEEE Trans. Broadcasting, vol. 52, no. 2, pp. 236-244, Jun. 2006.

[32] Z.-W. Zheng, Z.-X. Yang, C.-Y. Pan, and Y.-S. Zhu, "Novel synchronization for TDS-OFDM-based digital television terrestrial broadcast systems," IEEE Trans. Broadcasting, vol. 50, no. 2, pp. 148-153, Jun. 2004.

[33] J.-T. Wang, J. Song, J. Wang, C.-Y. Pan, Z.-X. Yang, and L. Yang, "A general SFN structure with transmit diversity for TDS-OFDM system," IEEE Trans. Broadcasting, vol. 52, no. 2, pp. 245-251, Jun. 2006.

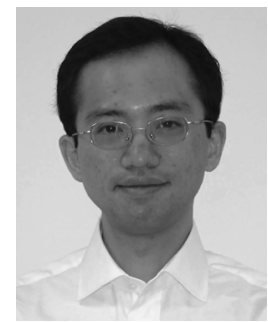

Hao Zhou was born in Zhejiang, China, in 1974. He received the B.S. and M.S. degrees in Electronic Engineering Department from SJTU, Shanghai, China, in 1997 and 2000. He studied at University of Notre Dame, IN since 2001 and received the M.S. and $\mathrm{Ph} . \mathrm{D}$. degrees in the department of Electrical Engineering from University of Notre Dame, in 2003 and 2007. From 2007, he is a senior signal processing engineer with Atheros Communications, Santa Clara, CA. His current research interests include mobile communications, satellite communications, modulation/demodulation, coding/decoding, and digital signal processing.

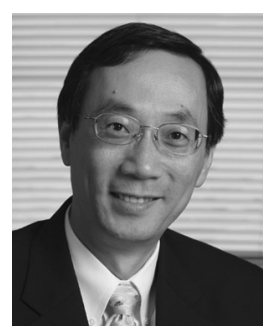

Yih-Fang Huang (F'95) is Professor of the Department of Electrical Engineering at University of Notre Dame where he started as an assistant professor upon receiving his Ph.D. in 1982. He served as chair of the department from 1998 to 2006. In Spring 1993, Dr. Huang received the Toshiba Fellowship and was Toshiba Visiting Professor at Waseda University, Tokyo, Japan, in the Department of Electrical Engineering. From April to July 2007, he was a visiting professor at the Technical University of Munich. In Fall, 2007, Dr. Huang was awarded the Fulbright-Nokia scholarship for lectures/research at Helsinki University of Technology.

Dr. Huang's research interests focus on statistical and adaptive signal processing. He has contributed to the field of Set-Membership Filtering (SMF), having developed a group of adaptive algorithms known as optimal bounding ellipsoids (OBE) algorithms. His current interests are in applying statistical/adaptive signal processing to multiple-access wireless communication systems.

Dr. Huang received the Golden Jubilee Medal of the IEEE Circuits and Systems Society in 1999, served as Vice President in 1997-98 and was a Distinguished Lecturer for the same society in 2000-2001. He received the University of Notre Dame's Presidential Award in 2003. Dr. Huang is a Fellow of the IEEE. 